\title{
GROWTH on S190426c: Real-time Search for a Counterpart to the Probable Neutron Star-Black Hole Merger using an Automated Difference Imaging Pipeline for DECam
}

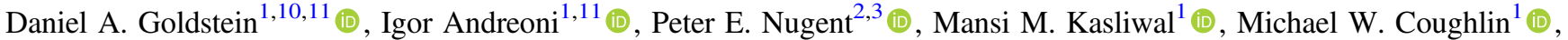 \\ Shreya Anand ${ }^{1}$, Joshua S. Bloom ${ }^{3,4}$, Jorge Martínez-Palomera ${ }^{3}$, Keming Zhang (张可名) ${ }^{3}$, Tomás Ahumada ${ }^{5}$, Ashot Bagdasaryan ${ }^{1}$, \\ Jeff Cooke ${ }^{6,7}$, Kishalay De $^{1}$, Dmitry A. Duev ${ }^{1}$ (i) , U. Christoffer Fremling ${ }^{1}$ (iD), Pradip Gatkine ${ }^{5}$ (D), Matthew Graham ${ }^{1}$ (D), \\ Eran O. Ofek ${ }^{8}$ (10), Leo P. Singer ${ }^{9}$ (1), and Lin Yan $^{1}$ (1) \\ ${ }^{1}$ California Institute of Technology, 1200 East California Boulevard, MC 249-17, Pasadena, CA 91125, USA \\ ${ }^{2}$ Computational Science Department, Lawrence Berkeley National Laboratory, 1 Cyclotron Road, MS 50B-4206, Berkeley, CA 94720, USA \\ ${ }^{3}$ Department of Astronomy, University of California, Berkeley, 501 Campbell Hall \#3411, Berkeley, CA 94720, USA \\ ${ }^{4}$ Lawrence Berkeley National Laboratory, 1 Cyclotron Road MS 50B-4206, Berkeley, CA 94720, USA \\ ${ }^{5}$ Department of Astronomy, University of Maryland, College Park, MD 20742, USA \\ ${ }^{6}$ Australian Research Council Centre of Excellence for Gravitational Wave Discovery (OzGrav), Swinburne University of Technology, Hawthorn, VIC 3122, \\ Australia \\ ${ }^{7}$ Centre for Astrophysics and Supercomputing, Swinburne University of Technology, Hawthorn, VIC 3122, Australia \\ ${ }^{8}$ Department of Particle Physics \& Astrophysics, Weizmann Institute of Science, Rehovot 76100, Israel \\ ${ }^{9}$ Goddard Space Flight Center, 8800 Greenbelt Road, Greenbelt, MD 20771, USA \\ Received 2019 May 16; revised 2019 July 2; accepted 2019 July 6; published 2019 August 5
}

\begin{abstract}
The discovery of a transient kilonova following the gravitational-wave (GW) event GW170817 highlighted the critical need for coordinated rapid and wide-field observations, inference, and follow-up across the electromagnetic spectrum. In the southern hemisphere, the Dark Energy Camera (DECam) on the Blanco $4 \mathrm{~m}$ telescope is well suited to this task, as it is able to cover wide fields quickly while still achieving the depths required to find kilonovae like the one accompanying GW170817 to 500 Mpc, the binary neutron star (NS) horizon distance for current generation of LIGO/Virgo collaboration (LVC) interferometers. Here, as part of the multi-facility followup by the Global Relay of Observatories Watching Transients Happen collaboration, we describe the observations and automated data movement, data reduction, candidate discovery, and vetting pipeline of our target-ofopportunity DECam observations of S190426c, the first possible NS-black hole merger detected in GWs. Starting $7.5 \mathrm{hr}$ after S190426c, over $11.28 \mathrm{hr}$ of observations, we imaged an area of $525 \mathrm{deg}^{2}\left(r\right.$ band) and $437 \mathrm{deg}^{2}(z$ band); this was $16.3 \%$ of the total original localization probability, and nearly all of the probability visible from the southern hemisphere. The machine-learning-based pipeline was optimized for fast turnaround, delivering transients for human vetting within 17 minutes, on average, of shutter closure. We reported nine promising counterpart candidates $2.5 \mathrm{hr}$ before the end of our observations. One hour after our data-taking ended (roughly $20 \mathrm{hr}$ after the announcement of S190426c), LVC released a refined skymap that reduced the probability coverage of our observations to $8.0 \%$, demonstrating a critical need for localization updates on shorter ( $\sim$ hour) timescales. Our observations yielded no detection of a bona fide counterpart to $m_{z}=21.7$ and $m_{r}=22.2$ at the $5 \sigma$ level of significance, consistent with the refined LVC positioning. We view these observations and rapid inferencing as an important real-world test for this novel end-to-end wide-field pipeline.
\end{abstract}

Key words: gravitational waves - stars: black holes - stars: neutron - surveys

\section{Introduction}

Joint detections of electromagnetic (EM) and gravitational waves (GWs) from compact binary mergers involving neutron stars (NSs) are a promising new way to address a number of open questions in astrophysics and cosmology (see, e.g., Bloom et al. 2009; Cowperthwaite et al. 2019a for reviews). The combined EM/GW data set from the binary neutron star (BNS) merger GW170817 (Abbott et al. 2017c) provided a high-precision measurement of the speed of gravity (Abbott et al. 2017b), gave new insight into the origin of the heavy elements (e.g., Chornock et al. 2017; Coulter et al. 2017; Drout et al. 2017; Evans et al. 2017; Kasen et al. 2017; Pian et al. 2017; Smartt et al. 2017; Côté et al. 2018, 2019; Siegel et al. 2019; Ji et al. 2019; Kasliwal et al. 2019a; Wu et al. 2019), demonstrated a novel technique for measuring cosmological

\footnotetext{
${ }^{10}$ Hubble Fellow.

11 These authors contributed equally to this work.
}

parameters (Abbott et al. 2017a), and provided unparalleled insight into the radiation hydrodynamics of compact binary mergers (e.g., Alexander et al. 2017; Haggard et al. 2017; Hallinan et al. 2017; Kasliwal et al. 2017; Margutti et al. 2017; Troja et al. 2017; D'Avanzo et al. 2018; Lazzati et al. 2018; Mooley et al. 2018; Ghirlanda et al. 2019). To date, GW170817 remains the only astrophysical event that has been detected in both the EM and GW messengers. To realize the full scientific potential of BNS and NS-black hole (BH) mergers with joint EM/GW detections, many more must be discovered and followed up.

The current working procedure for joint EM/GW astronomy begins when a network of GW observatories-presently, the Laser Interferometer Gravitational-Wave Observatory (LIGO; LIGO Scientific Collaboration et al. 2015) and the Virgo Gravitational-Wave Observatory (Acernese et al. 2015)detect a GW source, and, by analyzing its waveform, localizes it to a region of the sky that is typically between 100 and 1000 $\mathrm{deg}^{2}$. Nearly contemporaneous $\gamma$-rays and X-rays may be 
detected and localized if the merger also produces a short gamma-ray burst (GRB) at a favorable viewing angle (see, e.g., Eichler et al. 1989; Bloom et al. 2006). It then falls to the optical and near-infrared observational communities to search for transient events in the large localization regions that are consistent with theoretical expectations for spectrum synthesis in compact binary mergers, enabling the GW sources to be localized precisely (i.e., associated with a host galaxy). Such transients, often referred to as "kilonovae" because they are roughly $10^{3}$ times brighter than novae, are powered by the rapid decay of $r$-process material synthesized in the mergers (Metzger et al. 2010), and they are distinguished from other transients by their rapidly evolving light curves, which fade and redden in just a few days (e.g., Barnes \& Kasen 2013; Tanaka $\&$ Hotokezaka 2013). In order to search large areas of sky for such faint and rapidly evolving transients, telescopes with large apertures, imagers with large fields of view, and pipelines that can rapidly process images to efficiently identify transient candidates are required.

In the southern hemisphere, the Dark Energy Camera (DECam; Flaugher et al. 2015) on the Victor M. Blanco $4 \mathrm{~m}$ Telescope at Cerro Tololo Inter-American Observatory (CTIO) is particularly well suited to this task. The wide field of view $\left(\sim 3 \mathrm{deg}^{2}\right)$ of the instrument, combined with its red sensitivity and the substantial aperture of its telescope, make it well suited to follow up even the most distant BNS and NS-BH mergers in the horizon of the current era of LIGO/Virgo interferometers. The power of DECam for EM/GW follow-up was illustrated by its significant role in the study of AT 2017gfo, the kilonova associated with GW170817 (Cowperthwaite et al. 2017; Soares-Santos et al. 2017b), and by its important role in the follow-up of several other GW events from LIGO and Virgo (Annis et al. 2016; Cowperthwaite et al. 2016; Soares-Santos et al. 2016; Doctor et al. 2019).

In preparation for the third LIGO/Virgo GW observing run (O3), we developed a high-performance image subtraction pipeline to rapidly identify transients on DECam images. The National Optical Astronomy Observatory (NOAO), which allocates time on DECam, granted our team the opportunity to trigger the instrument to follow up NS mergers detected in GWs by LIGO and Virgo during the first half of O3 (NOAO Proposal ID 2019A-0205; PIs: Goldstein and Andreoni). We activated our first trigger on the unusual GW source S190426c (LIGO Scientific Collaboration and Virgo Collaboration 2019b), potentially the first NS-BH merger to be detected by LIGO and Virgo. In this Letter, we describe our follow-up observations of this event, with a focus on the software infrastructure we have developed to rapidly conduct wide-field optical follow-up observations of NS mergers using DECam. We describe additional Global Relay of Observatories Watching Transients Happen (GROWTH) searches for the electromagnetic counterpart to S190426c using the Zwicky Transient Facility, the GROWTH-India telescope, and an array of spectroscopic facilities in a suite of companion papers (V. B. Bhalerao et al. 2019, in preparation; M. M. K. Kasliwal et al. 2019, in preparation). A synopsis of the worldwide community observations reported in GCNs can be found in Hosseinzadeh et al. (2019).

\section{S190426c: A Probable NS-BH Merger}

On 2019 April 26 at 15:21:55 UTC, the LIGO Scientific Collaboration and Virgo Collaboration (LVC) identified a compact binary merger candidate, dubbed "S190426c," during real-time processing of data from LIGO Hanford Observatory, LIGO Livingston Observatory, and Virgo Observatory. The candidate was detected by four separate analysis pipelines: GstLAL (Messick et al. 2017), MBTAOnline (Adams et al. 2016), PyCBC Live (Nitz et al. 2017), and SPIIR, with a falsealarm rate of 1 in $1.7 \mathrm{yr}$. Roughly 20 minutes after detecting the event, LVC issued a circular on the NASA Gamma-Ray Coordinates Network $(\mathrm{GCN})^{12}$ reporting the discovery (LIGO Scientific Collaboration and Virgo Collaboration 2019a).

The initial GCN included a preliminary skymap giving a probabilistic localization of the event from the BAYESTAR rapid GW localization code (Singer \& Price 2016; see Figure 1). The total area of sky covered by the $90 \%$ confidence region was $1262 \mathrm{deg}^{2}$, with an estimated luminosity distance of $375 \pm 108 \mathrm{Mpc}$. As Figure 1 shows, the probability was concentrated in two distinct regions on the sky, one largely north of the celestial equator at R.A. $\approx 20.5$, and another region south of the equator roughly centered at R.A. $\approx 13.5$. On 2019 April 27 at 11:27:08 UTC, LVC issued a GCN containing a refined skymap from the LALInference GW localization code (Veitch et al. 2015) that localized the GW source to an area of $1131 \mathrm{deg}^{2}$ (90\% confidence), eliminating most of the probability in the southern hemisphere (LIGO Scientific Collaboration and Virgo Collaboration 2019d).

The initial classification of the event was consistent with several possible progenitor scenarios. The initial GCN circular classified the event as a BNS merger with a probability of $49 \%$, a compact binary merger with at least one object with a mass in the hypothetical "mass gap" between NSs and black holes (3-5 solar masses) with a probability of $24 \%$, a terrestrial event (i.e., not astrophysical) with a probability of $14 \%$, and an NS-BH merger with a probability of $13 \%$ (LIGO Scientific Collaboration and Virgo Collaboration 2019b). These probabilities were later updated in favor of the NS-BH interpretation, which was assigned a revised probability of $73.1 \%$ (including the mass gap probability), with no change to the probability of being a terrestrial event (LIGO Scientific Collaboration and Virgo Collaboration 2019c). Given the significant probability of the event originating from an NS merger, we decided to trigger our DECam program to search for an optical counterpart.

\section{Observations}

We triggered DECam follow-up of S190426c under NOAO proposal 2019A-0205 (PIs: Goldstein \& Andreoni), publishing a GCN circular describing our plan for the observations (Goldstein et al. 2019) and our intentions to make the data public immediately. We adopted an integrated observing strategy using the $r$ and $z$ filters with $30 \mathrm{~s}$ and $50 \mathrm{~s}$ exposures, respectively. The visits in $r$ and $z$ were spaced in time by at least 30 minutes to facilitate the rejection of moving objects. We observed from 2019 April 26 22:57:35 (about 7.5 hr postmerger) until 2019 April 27 10:25:54 UT, for a total of $11.28 \mathrm{hr}$. We acquired 196 exposures in $r$ and 163 in $z$, covering an area of $525 \mathrm{deg}^{2}$ and $437 \mathrm{deg}^{2}$, respectively, assuming an effective 60-CCD $2.68 \mathrm{deg}^{2}$ field of view for DECam that excludes the chip gaps. The observations covered $16.3 \%$ of the total original localization probability and $8.0 \%$ of the refined probability. Our observations resulted in empirical

\footnotetext{
$\overline{12 \text { https://gcn.gsfc.nasa.gov/gcn3_archive.html }}$
} 


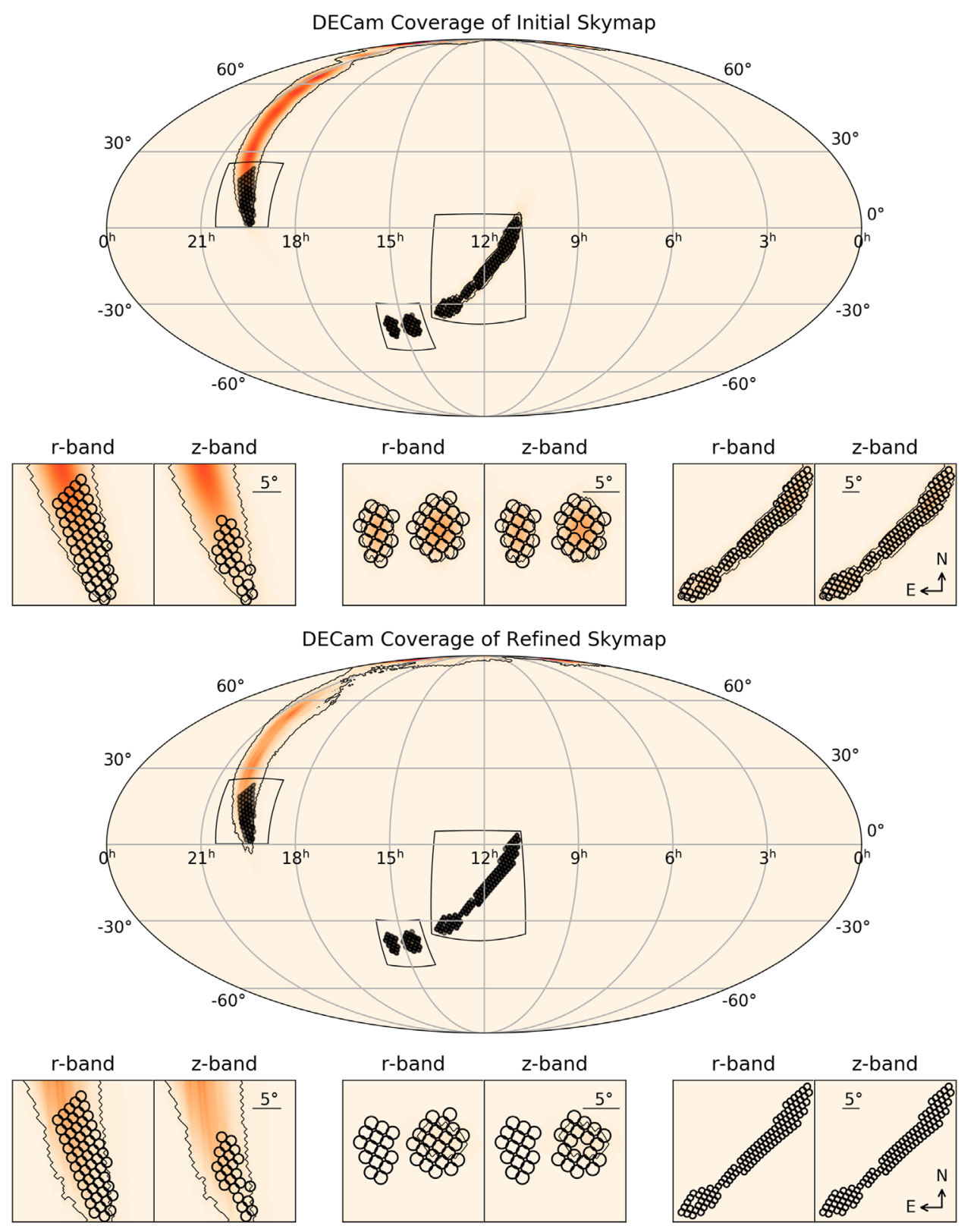

Figure 1. Coverage of the initial (BAYESTAR) and refined (LALInference) skymaps for S190426c by the observations presented in this Letter. Each black circle represents one DECam pointing, where the $3 \mathrm{deg}^{2}$ DECam field of view has been approximated as a circle of radius 0.9 . Nearly uniform coverage of the region of the initial skymap visible from CTIO was obtained in the $r$ and $z$ bands. In total, 16.3\% of the BAYESTAR probability was enclosed by the observations. This probability dropped to $8.0 \%$ when the refined LALInference skymap was released.

limiting magnitudes of $m_{z}=21.7$ and $m_{r}=22.2$ at the $5 \sigma$ level of significance.

The information provided by the initial LVC skymap (large localization area, large distance, possible $\mathrm{BH}$ companion) compelled us to modify the observing strategy that we originally designed for this program, which was based on three visits in $g-z-g$ bands on the first night and a $g-z$ pair on the second night after the trigger. Exposure times were planned to be $15 \mathrm{~s}$ in $g$ and $25 \mathrm{~s}$ in the $z$ band. Such a strategy was designed to follow up primarily BNS mergers enclosed in an error region $\lesssim 150 \mathrm{deg}^{2}$ in extension and $<200 \mathrm{Mpc}$ away. The $g-z$ filter combination is well suited to capture and recognize the rapidly evolving blue component that BNS mergers such as GW170817 have been observed to show (see, e.g., Evans et al. 2017; Shappee et al. 2017; Andreoni et al. 2019a;
Cowperthwaite et al. 2019b). The large distance to S190426c, along with the theoretical expectation that NS-BH mergers may not show any bright blue component at early times (Kasen et al. 2017), advocated in favor of deeper exposures and redder filters. The third visit planned for the first night was dropped in favor of a broader sky coverage with longer $z$-band exposures. For further details on schedule optimization for our DECam program, see Andreoni et al. (2019c).

Our observations of S190426c were scheduled automatically by the GROWTH target-of-opportunity (ToO) marshal system $^{13}$ described in Coughlin et al. (2019) and Kasliwal et al. (2019b). For this event, we instructed the ToO marshal to

$\overline{{ }^{13} \text { https://github.com/growth-astro/growth-too-marshal }}$ 
employ a "greedy" algorithm to generate a schedule of observations that tiled as much of the $90 \%$ credible position region of the initial skymap as possible. The schedule was generated before sunset in Chile on 2019 April 26 and exported as a json file. The initial skymap and our series of observations are shown in Figure 1. The json file was ingested into the DECam Survey Image System Process Integration (SISPI; Honscheid et al. 2012) readout and control system, which executed the observations. As soon as each exposure was completed, SISPI transferred each raw exposure to NOAO in Tucson, AZ, via the Data Transport System (DTS; Fitzpatrick 2010) for archiving.

A second epoch was planned for the following night using the same filters, but the refined skymap that LVC released about $1 \mathrm{hr}$ after our observations (roughly $20 \mathrm{hr}$ postmerger; LIGO Scientific Collaboration and Virgo Collaboration 2019d) using the more precise LALInference localization pipeline (Veitch et al. 2015) eliminated the localization probability in any sky region with template coverage (see Section 4.3). Moreover, the visible region of sky that we could have observed resides on the Galactic plane, where several magnitudes of extinction and crowded stellar fields make the detection of faint, extragalactic transients difficult. Therefore, we decided against more disruptive ToO observations, ending our DECam observing campaign for S190426c after a single night of data-taking. If the refined LALInference skymap had been released 4-5 hr earlier, we could have conserved roughly half a night of DECam time and focused our observations on the regions of sky that remained consistent with the GW signal. Valuable telescope resources could be better optimized with faster localization updates from LVC.

\section{Real-time Automated Difference Imaging Pipeline}

As soon as observations commenced on the first night of our trigger, we programmatically checked the NOAO archive each second for new images from the DTS. Each time a new image was found, we automatically downloaded it over FTP to the National Energy Research Scientific Computing Center (NERSC) in Berkeley, CA, and stored it on a high-performance Lustre parallel filesystem, making use of the ESNet energy sciences high-speed Internet backbone that connects national laboratories, universities, and other research institutions. The typical data transfer rate from Tucson to Berkeley was $40 \mathrm{MB} \mathrm{s}^{-1}$, enabling each $550 \mathrm{MB}$ fits focal plane exposure to be delivered in an average transfer time of $14 \mathrm{~s}$.

\subsection{Exposure Segmentation and Parallelization}

When each raw image arrived at NERSC, a job was programmatically launched via $\mathrm{slurm}^{14}$ to process it, beginning the real-time search. Jobs were executed on the Cray XC40 cori supercomputer. Each exposure was delegated for processing to a single 64-logical core haswell compute node. In each job, each of the 62 DECam science CCDs was assigned to a single logical core. We arranged a special, low-latency "real-time" job queue for this project to provide nearimmediate access to NERSC computing resources. Our realtime queue gave us on-demand access to 18 haswell compute nodes, allowing us to process up to 18 exposures

\footnotetext{
${ }^{14}$ https://slurm.schedmd.com/overview.html
}

simultaneously. We found that this allocation of computing resources was sufficient to ensure fast turnaround.

As a first step in the processing, each raw DECam fits file was split into 62 separate fits files, one for each CCD. Except for template generation, all subsequent pipeline steps were performed on a per-CCD basis, using the Message Passing Interface (MPI) to facilitate the concurrent execution of 62 independent copies of the pipeline in each of up to 18 jobs running simultaneously. The top-level pipeline code was written in the Python programming language and run inside a high-performance shifter ${ }^{15}$ container to increase performance on the NERSC hardware.

\subsection{Detrending and Astrometric Calibration}

The raw frames we ingested from the NOAO archive contained only fits header keywords, a rough world coordinate system solution, and integer pixel values, so we first performed a series of detrending and preprocessing steps to transform them into usable science frames using custom preprocessing software. For each frame, we made an overscan correction as described in Bernstein et al. (2017). We also generated a mask frame for each CCD, masking out any pixels above the saturation value of their amplifier. Because our observations were time sensitive, and because DECam is a very stable instrument, we used flat and bias frames from a previous night for the real-time processing (i.e., we did not take flats or bias frames in our observing sequence). The flat and bias frames we used to process the data for S190426c were taken on 2018 November 1 as part of the DECam Legacy Survey (Dey et al. 2019). We subtracted the bias frames from the raw pixels and then divided by the flat frames. Any science pixel values rendered invalid by the flat-fielding were masked. We applied the standard DECam bad pixel masks, but to achieve fast turnaround did not apply cross-talk corrections or correct for the brighter-fatter effect. For DECam, the effect of cross talk on measured fluxes is at the $0.1 \%$ level between intra-CCD amplifier pairs, and at the $0.01 \%$ level between CCDs (Bernstein et al. 2017), and the brighter-fatter effect is even smaller (Gruen et al. 2015). These effects are thus safely subdominant to those of photometric calibration and photon statistics, which give uncertainties at the few to ten percent level for most of the sources here. We processed all science CCDs from each pointing, including those that have been deemed defective (N30 and $\mathrm{S} 30$ ). We produced a source catalog of each detrended science image using SExtractor (Bertin \& Arnouts 1996) that we fed into a developmentbranch version of SCAMP (Bertin 2006) to perform astrometric calibration against the Gaia DR1 catalog (Gaia Collaboration et al. 2016). The average absolute astrometric uncertainty of the solutions with respect to Gaia DR1 was 100 mas.

\subsection{Template Generation}

To perform image subtraction, we assembled a library of template images from three publicly available DECam data sets: the Dark Energy Survey DR1 (Dark Energy Survey Collaboration et al. 2016; Abbott et al. 2018), the DECam Legacy Survey DR7 (Dey et al. 2019), and the Blanco Imaging of the Southern Sky Survey (BLISS; Soares-Santos et al. 2017a) stacked images distributed by the NOAO archive. We

\footnotetext{
${ }_{15}$ A docker-like containerization service for high-performance computing, see Gerhardt et al. (2017).
} 

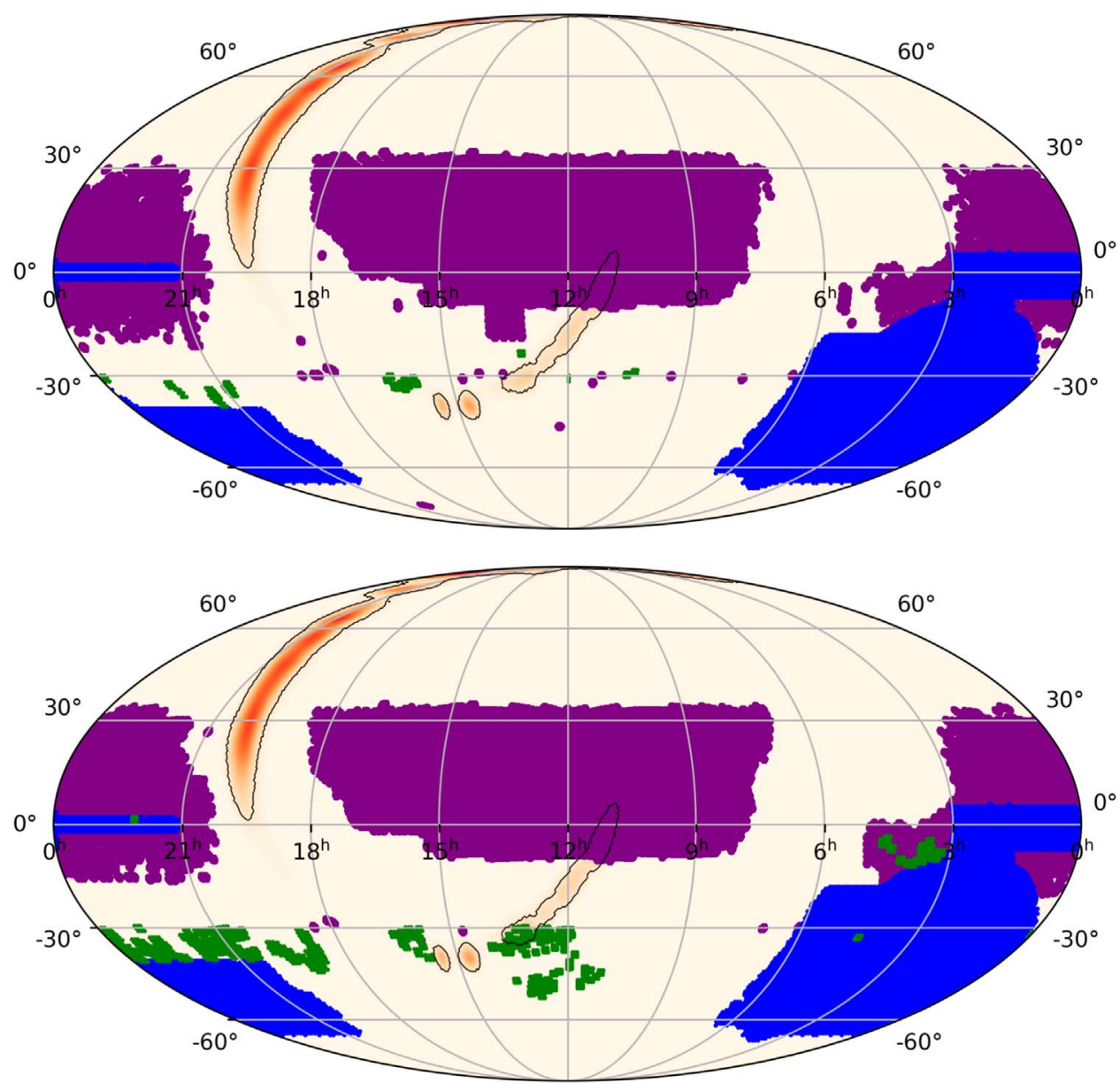

Figure 2. Accumulated $r$-band (top) and $z$-band (bottom) DECam template coverage of our pipeline, plotted against the initial skymap of S190426c, which set our observing strategy. Our template bank drew from DES (blue), DECaLS (purple), and BLISS (green) images.

downloaded all of these astrometrically and photometrically calibrated template images to disk at NERSC. In total, the templates required about $50 \mathrm{~TB}$ of disk space and covered about $14,500 \mathrm{deg}^{2}$ of the sky below a decl. of $+30^{\circ}$. Our $r$ - and $z$-band template coverage relative to the initial skymap of S190426c is shown in Figure 2. Only a small region of the skymap for this event had template coverage (about $120 \mathrm{deg}^{2}$ in the $z$ band, and $100 \mathrm{deg}^{2}$ in the $r$ and $z$ bands) from the DECaLS and BLISS surveys. We used SWarp (Bertin 2010) to combine and crop the individual template images into references for each CCD. The coaddition employed clipped mean stacking to suppress artifacts and increase signal to noise (Gruen et al. 2014). The pipeline produced template images on the fly for each CCD and pointing. For images with no template coverage, the pipeline exited gracefully. We are currently working to improve the template coverage of our pipeline by integrating more exposures that are publicly available from the NOAO archive.

\subsection{Photometric Calibration}

To photometrically calibrate our science images, we compared the magnitudes of stars extracted with SExtractor to the same stars on the reference images. We then derived a zero-point for the science images by taking the median zeropoint derived from each calibrator. We also used this procedure to estimate the seeing on the science images, taking the median FWHM of each calibrator. To choose calibrators, we selected only objects with no SExtractor extraction error flags and a signal-to-noise ratio of at least 5 . We performed all photometry via point-spread function (PSF) fitting, constructing a spatially varying PSF model using the PSFEx software.

\subsection{Image Subtraction, Source Identification, and Artifact Rejection}

For each pair of photometrically and astrometrically calibrated science images and templates, we used scamp to align the images to a common $x-y$ grid and the HOTPANTS (Becker 2015) implementation of the Alard \& Lupton (1998) algorithm to convolve the images to a common PSF and perform a pixel-by-pixel subtraction. We then ran SExtractor on the resulting difference images to identify sources of variability. We rejected any objects that overlapped masked pixels on either the template or science images, had SExtractor extraction flags, had an axis ratio greater than 1.5, had an FWHM more than twice the seeing, had a PSF magnitude greater than 30 , had a signal-to-noise ratio less than 5 , or had a semimajor axis less than 1 pixel. After making these initial cuts, we used the publicly available autoScan code (Goldstein et al. 2015), based on the machine-learning technique Random Forest, to probabilistically classify the "realness" of the remaining extracted sources. The code has been successfully used in past DECam searches for GW counterparts in independent difference imaging pipelines (e.g., Soares-Santos et al. 2017b). 
new
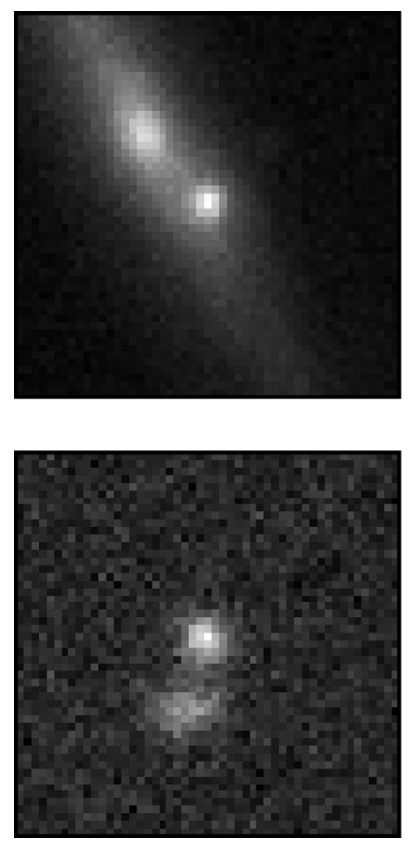

ref
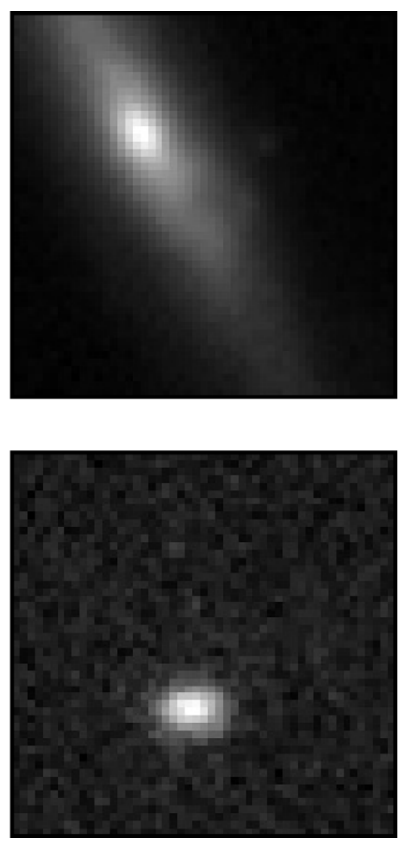

sub
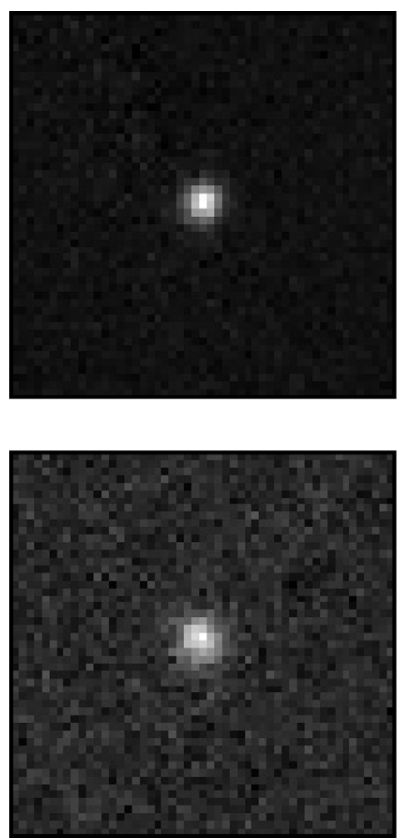

ps1
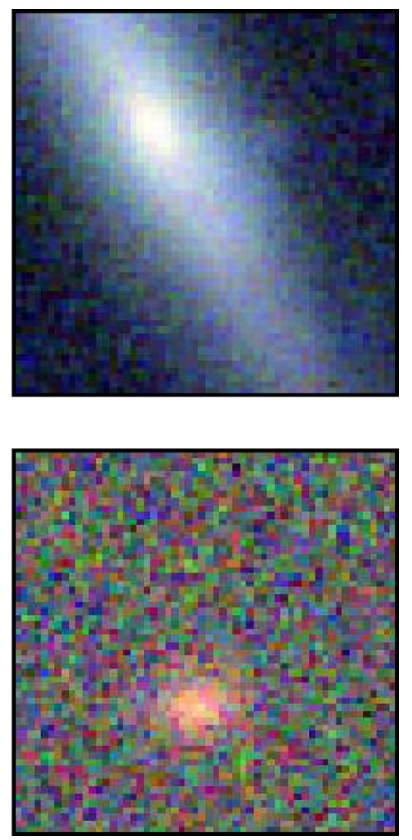

Figure 3. Postage-stamp cutouts of some transients identified by the pipeline (top: DG19vkgf, bottom: DG19ytre). Each transient has at least one detection in both $r$ and $z$, separated by at least 30 minutes (to reject asteroids). Full color images from Pan-STARRS1 (PS1) are shown for reference.

Table 1

Candidates Discovered in Real Time Using our Transient Detection Pipeline and Reported in Andreoni et al. (2019b)

\begin{tabular}{|c|c|c|c|c|c|c|}
\hline Name & $\begin{array}{l}\text { R.A. } \\
\text { (J2000) }\end{array}$ & $\begin{array}{l}\text { Decl. } \\
\text { (J2000) }\end{array}$ & Filter & $m$ & $\sigma_{m}$ & MJD \\
\hline DG19ftnb & 167.595555 & -4.358792 & $\begin{array}{l}z \\
r\end{array}$ & $\begin{array}{l}19.31 \\
19.811\end{array}$ & $\begin{array}{l}0.080 \\
0.077\end{array}$ & $\begin{array}{l}58599.99056 \\
58599.96644\end{array}$ \\
\hline DG19kqxe & 163.781705 & -0.237631 & $\begin{array}{l}z \\
r\end{array}$ & $\begin{array}{l}20.571 \\
21.297\end{array}$ & $\begin{array}{l}0.132 \\
0.131\end{array}$ & $\begin{array}{l}58600.17142 \\
58600.13044\end{array}$ \\
\hline DG19ouub & 171.473410 & -9.488396 & $\begin{array}{l}z \\
r\end{array}$ & $\begin{array}{l}20.077 \\
20.903\end{array}$ & $\begin{array}{l}0.108 \\
0.140\end{array}$ & $\begin{array}{l}58600.00142 \\
58599.97506\end{array}$ \\
\hline DG19vkgf & 165.844300 & -7.917442 & $\begin{array}{l}z \\
r\end{array}$ & $\begin{array}{l}18.78 \\
19.258\end{array}$ & $\begin{array}{l}0.02 \\
0.017\end{array}$ & $\begin{array}{l}58600.19049 \\
58600.15045\end{array}$ \\
\hline DG19zyaf & 163.471788 & -1.151129 & $\begin{array}{l}z \\
r\end{array}$ & $\begin{array}{l}21.181 \\
22.165\end{array}$ & $\begin{array}{l}0.12 \\
0.152\end{array}$ & $\begin{array}{l}58600.17142 \\
58600.13044\end{array}$ \\
\hline DG19pklb & 168.658618 & -6.975466 & $\begin{array}{l}z \\
r\end{array}$ & $\begin{array}{l}20.532 \\
19.33\end{array}$ & $\begin{array}{l}0.146 \\
0.09\end{array}$ & $\begin{array}{l}58599.99355 \\
58599.96570\end{array}$ \\
\hline DG19ytre & 167.760365 & 0.527199 & $\begin{array}{l}z \\
r\end{array}$ & $\begin{array}{l}20.828 \\
19.824\end{array}$ & $\begin{array}{l}0.087 \\
0.040\end{array}$ & $\begin{array}{l}58600.11954 \\
58600.08185\end{array}$ \\
\hline
\end{tabular}

Note. The magnitudes $(m)$ and uncertainties $\left(\sigma_{m}\right)$ are in the AB system. The midpoint observing time is given in Modified Julian days (MJD). Valeev et al. (2019) classified DG19pklb as a broad-line SN Ic at redshift $z=0.09123$ and DG19ytre as an SN Ia at $z=0.1386$, ruling out their association with S190426c.

We pushed the candidates immediately and automatically to the GROWTH marshal, a dynamic web portal for time-domain astronomy (Kasliwal et al. 2019b), where they were scanned by a team of roughly 10 scientists. We reported nine promising counterpart candidates via GCN $2.5 \mathrm{hr}$ before the end of our observations (Andreoni et al. 2019b). We used the numerical score assigned by autoScan to each candidate to determine the order in which we looked at objects. Using autoScan we were able to identify the transients we reported in the GCN by looking at less than $1 \%$ of the candidate pool. We also crossmatched each of our candidates against Gaia DR2 (Gaia
Collaboration et al. 2018) to reject variable stars, the Minor Planet Center online checker ${ }^{16}$ to reject asteroids, and the Transient Name Server ${ }^{17}$ to reject known transients. Figure 3 shows images of two example candidates identified by the pipeline that were reported in the GCN, and Table 1 gives DECam photometry of all candidates.

\footnotetext{
${ }_{16}$ https://minorplanetcenter.net/cgi-bin/checkmp.cgi

17 https://wis-tns.weizmann.ac.il/
} 

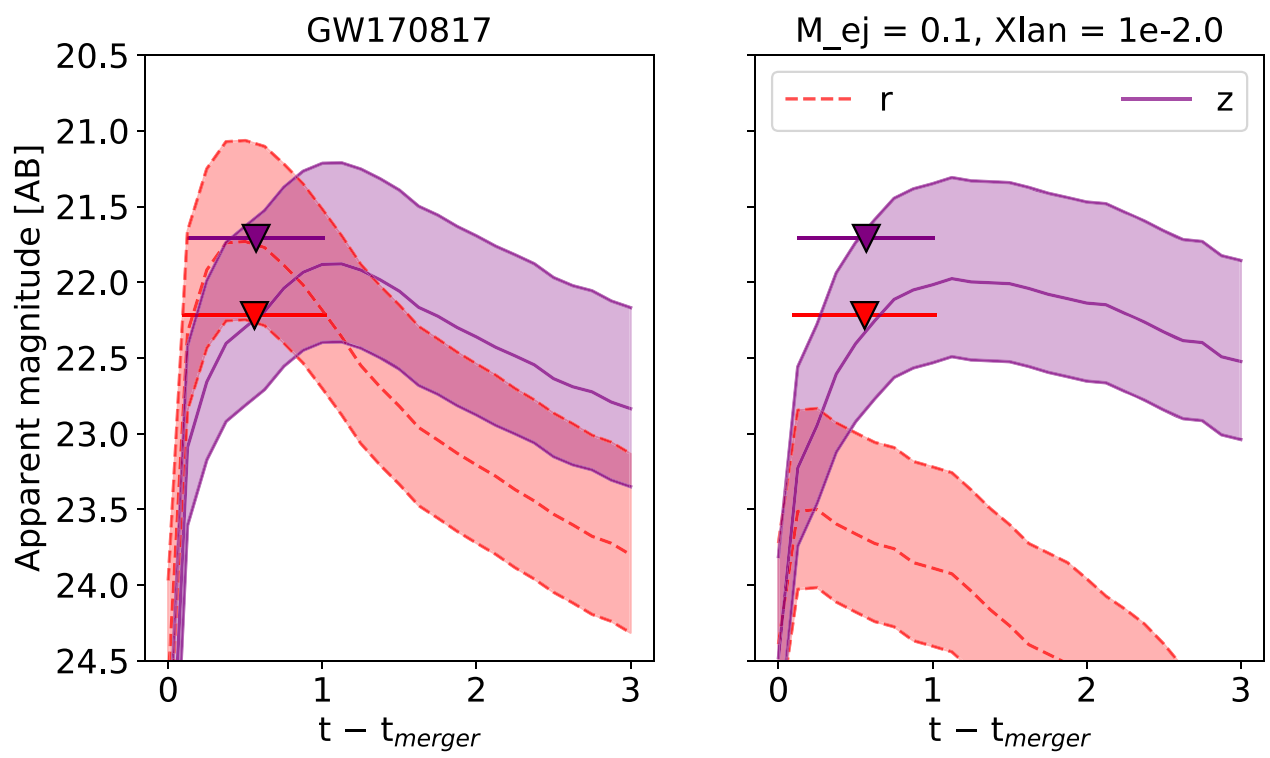

Figure 4. Two one-component kilonova models (Kasen et al. 2017) scaled to the distance range of S190426c (bands) plotted against the $5 \sigma$ detection limits of our $r$ and $z$-band observations (not corrected for Galactic extinction) in the 8\% of the localization region remaining after the release of the refined skymap. The left panel shows the light curve of the blue component of the BNS merger GW170817; the right panel presents a model with high ejecta mass $\left(M_{\mathrm{ej}}=0.1 M_{\odot}\right)$ and large lanthanide content, a more plausible scenario in the case of an NS-BH merger.

\subsection{Search Results and Pipeline Performance}

Processing each exposure with the pipeline required 16.7 minutes of wall-clock time, on average. This fast turnaround time allowed us to detect transients quickly and rapidly communicate them to the community. We identified 84,007 candidates: 45,587 in $r$-band and 48,931 in $z$-band images. 15,432 of our candidates had at least 2 detections separated by at least 30 minutes to reject moving objects. The measured depth reached during our observations would have likely enabled the detection in the $r$ band of a GW170817-like event (Figure 4, left panel). Radiation transport modeling suggests that if S190426c was in fact the result of an NS-BH merger, it would likely have not been detected in the $r$ band in our observations (Figure 4, right panel) due to the redder expected colors of NS-BH counterparts compared to those of GW170817-like transients (Kasen et al. 2017). One mildly red transient $(r-z=0.3$ in DECam images), labeled DG19vkgf, was spectroscopically and photometrically followed up by our team using the Hale 200-inch telescope (P200) at Palomar observatory (De et al. 2019). A spectrum was obtained with the Double Beam Spectrograph (Oke \& Gunn 1982) on P200. Due to the high airmass and poor seeing conditions, the transient was not clearly identified in the trace, but the host redshift was confirmed to be $z=0.04$ using the host emission lines. Imaging with the Wafer Scale Imager for Prime (WASP) on P200 confirmed the presence of a point source at the transient location. The refined skymap, released after follow-up observations of DG19vkgf had been obtained, eliminated the possibility that the transient was associated with S190426c, so no further data were collected. We did not secure a definitive classification of the transient.

Valeev et al. (2019) followed up two events reported in Andreoni et al. (2019b), DG19pklb and DG19ytre. The authors performed photometric follow-up using the $1.5 \mathrm{~m}$ telescope at the Observatorio de Sierra Nevada (Spain) starting on 2019 April 27 20:53 UT and spectroscopic follow-up using the $10.4 \mathrm{~m}$ Gran Telescopio Canarias equipped with OSIRIS at La Palma (Spain) starting on 2019 April 27 21:40 UT. Those observations allowed Valeev et al. (2019) to classify DG19pklb as a broad-lined SN Ic at redshift $z=0.09123$ and DG19ytre as an SN Ia at $z=0.1386$. The association of DG19pklb or DG19ytre with S190426c was therefore excluded.

When LIGO released a refined skymap (LIGO Scientific Collaboration and Virgo Collaboration 2019d) that completely ruled out the possible association of DG19vkgf or any of the other transients we discovered using our pipeline with S190426c, we interrupted our photometric and spectroscopic follow-up of those sources. We then focused our follow-up efforts on transients discovered with northern hemisphere facilities that could access regions of higher localization probability (V. B. Bhalerao et al. 2019, in preparation; M. M. K. Kasliwal et al. 2019, in preparation).

\section{Conclusion}

We carried out follow-up observations of the LIGO/Virgo GW trigger S190426c with DECam. Using an automated difference imaging pipeline, we were able to rapidly search our data and publish candidates to the community before we completed our observations. Although we did not identify a counterpart with these observations, this enabled us to validate our DECam infrastructure for future events, demonstrating that we can readily trigger, observe, scan, and detect transients on timescales, sky areas, and magnitude limits relevant for the discovery of GW counterparts. Availability of updated LVC skymaps on an even shorter timescale would allow us to more prudently use our telescope resources. In the future, we expect DECam to continue its important role as a discovery engine for GW counterparts.

D.A.G. and I.A. gratefully acknowledge Kathy Vivas, Steve Heathcote, and the NOAO staff for facilitating these target-ofopportunity observations. This research used resources of the National Energy Research Scientific Computing Center (NERSC), a U.S. Department of Energy Office of Science User Facility operated under Contract No. DE-AC0205CH11231. D.A.G. acknowledges support from Hubble 
Fellowship grant HST-HF2-51408.001-A. Support for Program number $H S T$-HF2-51408.001-A is provided by NASA through a grant from the Space Telescope Science Institute, which is operated by the Association of Universities for Research in Astronomy, Incorporated, under NASA contract NAS5-26555. P.E.N. acknowledges support from the DOE through DE-FOA0001088, Analytical Modeling for Extreme-Scale Computing Environments. E.O.O. is grateful for support by a grant from the Israeli Ministry of Science, ISF, Minerva, BSF, BSF transformative program, and the I-CORE Program of the Planning and Budgeting Committee and The Israel Science Foundation (grant No. 1829/12). M.W.C. is supported by the David and Ellen Lee Postdoctoral Fellowship at the California Institute of Technology. J. S.B., J.M.-P., and K.Z. are partially supported by a Gordon and Betty Moore Foundation DataDriven Discovery grant. J.C. is supported in part by the Australian Research Council Centre of Excellence for Gravitational Wave Discovery (OzGrav), CE170100004.

This work was supported by the GROWTH (Global Relay of Observatories Watching Transients Happen) project funded by the National Science Foundation under PIRE grant No. 1545949. GROWTH is a collaborative project among California Institute of Technology (USA), University of Maryland College Park (USA), University of Wisconsin Milwaukee (USA), Texas Tech University (USA), San Diego State University (USA), University of Washington (USA), Los Alamos National Laboratory (USA), Tokyo Institute of Technology (Japan), National Central University (Taiwan), Indian Institute of Astrophysics (India), Indian Institute of Technology Bombay (India), Weizmann Institute of Science (Israel), The Oskar Klein Centre at Stockholm University (Sweden), Humboldt University (Germany), Liverpool John Moores University (UK), University of Sydney (Australia) and Swinburne University of Technology (Australia).

This project used public archival data from the Dark Energy Survey (DES). Funding for the DES Projects has been provided by the U.S. Department of Energy, the U.S. National Science Foundation, the Ministry of Science and Education of Spain, the Science and Technology Facilities Council of the United Kingdom, the Higher Education Funding Council for England, the National Center for Supercomputing Applications at the University of Illinois at Urbana-Champaign, the Kavli Institute of Cosmological Physics at the University of Chicago, the Center for Cosmology and Astro-Particle Physics at the Ohio State University, the Mitchell Institute for Fundamental Physics and Astronomy at Texas A\&M University, Financiadora de Estudos e Projetos, Fundação Carlos Chagas Filho de Amparo à Pesquisa do Estado do Rio de Janeiro, Conselho Nacional de Desenvolvimento Científico e Tecnológico and the Ministério da Ciência, Tecnologia e Inovação, the Deutsche Forschungsgemeinschaft, and the Collaborating Institutions in the Dark Energy Survey. The Collaborating Institutions are Argonne National Laboratory, the University of California at Santa Cruz, the University of Cambridge, Centro de Investigaciones Energéticas, Medioambientales y Tecnológicas-Madrid, the University of Chicago, University College London, the DESBrazil Consortium, the University of Edinburgh, the Eidgenössische Technische Hochschule (ETH) Zürich, Fermi National Accelerator Laboratory, the University of Illinois at UrbanaChampaign, the Institut de Ciències de l'Espai (IEEC/CSIC), the Institut de Física d'Altes Energies, Lawrence Berkeley National Laboratory, the Ludwig-Maximilians Universität
München and the associated Excellence Cluster Universe, the University of Michigan, the National Optical Astronomy Observatory, the University of Nottingham, The Ohio State University, the OzDES Membership Consortium, the University of Pennsylvania, the University of Portsmouth, SLAC National Accelerator Laboratory, Stanford University, the University of Sussex, and Texas A\&M University. Based in part on observations at Cerro Tololo Inter-American Observatory, National Optical Astronomy Observatory, which is operated by the Association of Universities for Research in Astronomy (AURA) under a cooperative agreement with the National Science Foundation.

The Legacy Surveys consist of three individual and complementary projects: the Dark Energy Camera Legacy Survey (DECaLS; NOAO Proposal ID 2014B-0404; PIs: David Schlegel and Arjun Dey), the Beijing-Arizona Sky Survey (BASS; NOAO Proposal ID \# 2015A-0801; PIs: Zhou $\mathrm{Xu}$ and Xiaohui Fan), and the Mayall z-band Legacy Survey (MzLS; NOAO Proposal ID \# 2016A-0453; PI: Arjun Dey). DECaLS, BASS, and MzLS together include data obtained, respectively, at the Blanco telescope, Cerro Tololo InterAmerican Observatory, National Optical Astronomy Observatory (NOAO); the Bok telescope, Steward Observatory, University of Arizona; and the Mayall telescope, Kitt Peak National Observatory, NOAO. The Legacy Surveys project is honored to be permitted to conduct astronomical research on Iolkam Du'ag (Kitt Peak), a mountain with particular significance to the Tohono O'odham Nation.

NOAO is operated by the Association of Universities for Research in Astronomy (AURA) under a cooperative agreement with the National Science Foundation.

\section{ORCID iDs}

Daniel A. Goldstein (ํ) https://orcid.org/0000-0003-3461-8661 Igor Andreoni (1) https://orcid.org/0000-0002-8977-1498 Peter E. Nugent (1) https://orcid.org/0000-0002-3389-0586 Mansi M. Kasliwal (i) https://orcid.org/0000-0002-5619-4938 Michael W. Coughlin (ib https://orcid.org/0000-00028262-2924

Dmitry A. Duev (1) https://orcid.org/0000-0001-5060-8733 U. Christoffer Fremling (i) https://orcid.org/0000-00024223-103X

Pradip Gatkine iㅏ https://orcid.org/0000-0002-1955-2230 Matthew Graham (i) https://orcid.org/0000-0002-3168-0139 Eran O. Ofek (1) https://orcid.org/0000-0002-6786-8774 Leo P. Singer (1) https://orcid.org/0000-0001-9898-5597 Lin Yan (1) https://orcid.org/0000-0003-1710-9339

\section{References}

Abbott, B. P., Abbott, R., Abbott, T. D., et al. 2017a, Natur, 551, 85 Abbott, B. P., Abbott, R., Abbott, T. D., et al. 2017b, ApJL, 848, L13 Abbott, B. P., Abbott, R., Abbott, T. D., et al. 2017c, ApJL, 848, L12 Abbott, T. M. C., Abdalla, F. B., Allam, S., et al. 2018, ApJS, 239, 18 Acernese, F., Agathos, M., Agatsuma, K., et al. 2015, CQGra, 32, 024001 Adams, T., Buskulic, D., Germain, V., et al. 2016, CQGra, 33, 175012 Alard, C., \& Lupton, R. H. 1998, ApJ, 503, 325

Alexander, K. D., Berger, E., Fong, W., et al. 2017, ApJL, 848, L21 Andreoni, I., Anand, S., Bianco, F. B., et al. 2019a, PASP, 131, 068004 Andreoni, I., Goldstein, D. A., Coughlin, M., \& Kasliwal, M. M. 2019b, GCN, 24268, 1

Andreoni, I., Goldstein, D. A., Anand, S., et al. 2019c, ApJL, in press (arXiv:1906.00806)

Annis, J., Soares-Santos, M., Berger, E., et al. 2016, ApJL, 823, L34

Barnes, J., \& Kasen, D. 2013, ApJ, 775, 18 
Becker, A. 2015, HOTPANTS: High Order Transform of PSF ANd Template Subtraction, Astrophysics Source Code Library, ascl:1504.004

Bernstein, G. M., Abbott, T. M. C., Desai, S., et al. 2017, PASP, 129, 114502

Bertin, E. 2006, in ASP Conf. Ser. 351, Astronomical Data Analysis Software and Systems XV, ed. C. Gabriel et al. (San Francisco, CA: ASP), 112

Bertin, E. 2010, SWarp: Resampling and Co-adding FITS Images Together, Astrophysics Source Code Library, ascl:1010.068

Bertin, E., \& Arnouts, S. 1996, A\&AS, 117, 393

Bloom, J. S., Holz, D. E., Hughes, S. A., et al. 2009, arXiv:0902.1527

Bloom, J. S., Prochaska, J. X., Pooley, D., et al. 2006, ApJ, 638, 354

Chornock, R., Berger, E., Kasen, D., et al. 2017, ApJL, 848, L19

Côté, B., Eichler, M., Arcones, A., et al. 2019, ApJ, 875, 106

Côté, B., Fryer, C. L., Belczynski, K., et al. 2018, ApJ, 855, 99

Coughlin, M. W., Ahumada, T., Cenko, S. B., et al. 2019, PASP, 131, 048001

Coulter, D. A., Foley, R. J., Kilpatrick, C. D., et al. 2017, Sci, 358, 1556

Cowperthwaite, P. S., Berger, E., Soares-Santos, M., et al. 2016, ApJL, 826, L29

Cowperthwaite, P. S., Berger, E., Villar, V. A., et al. 2017, ApJL, 848, L17

Cowperthwaite, P. S., Chen, H.-Y., Margalit, B., et al. 2019a, arXiv:1904. 02718

Cowperthwaite, P. S., Villar, V. A., Scolnic, D. M., \& Berger, E. 2019b, ApJ, 874, 88

Dark Energy Survey Collaboration, Abbott, T., Abdalla, F. B., et al. 2016, MNRAS, 460, 1270

D’Avanzo, P., Campana, S., Salafia, O. S., et al. 2018, A\&A, 613, L1

De, K., Yan, L., Fremling, C., \& Andreoni, I. 2019, GCN, 24275, 1

Dey, A., Schlegel, D. J., Lang, D., et al. 2019, AJ, 157, 168

Doctor, Z., Kessler, R., Herner, K., et al. 2019, ApJL, 873, L24

Drout, M. R., Piro, A. L., Shappee, B. J., et al. 2017, Sci, 358, 1570

Eichler, D., Livio, M., Piran, T., \& Schramm, D. N. 1989, Natur, 340, 126

Evans, P. A., Cenko, S. B., Kennea, J. A., et al. 2017, Sci, 358, 1565

Fitzpatrick, M. J. 2010, Proc. SPIE, 7737, 77371T

Flaugher, B., Diehl, H. T., Honscheid, K., et al. 2015, AJ, 150, 150

Gaia Collaboration, Brown, A. G. A., Vallenari, A., et al. 2016, A\&A, 595, A2

Gaia Collaboration, Brown, A. G. A., Vallenari, A., et al. 2018, A\&A, 616, A1

Gerhardt, L., Bhimji, W., Canon, S., et al. 2017, JPhCS, 898, 082021

Ghirlanda, G., Salafia, O. S., Paragi, Z., et al. 2019, Sci, 363, 968

Goldstein, D. A., Andreoni, I., Coughlin, M., \& Kasliwal, M. M. 2019, GCN, 24257, 1

Goldstein, D. A., D'Andrea, C. B., Fischer, J. A., et al. 2015, AJ, 150, 82

Gruen, D., Bernstein, G. M., Jarvis, M., et al. 2015, JInst, 10, C05032

Gruen, D., Seitz, S., \& Bernstein, G. M. 2014, PASP, 126, 158

Haggard, D., Nynka, M., Ruan, J. J., et al. 2017, ApJL, 848, L25
Hallinan, G., Corsi, A., Mooley, K. P., et al. 2017, Sci, 358, 1579

Honscheid, K., Elliott, A., Annis, J., et al. 2012, Proc. SPIE, 8451, 845112

Hosseinzadeh, G., Cowperthwaite, P. S., Gomez, S., et al. 2019, arXiv:1905. 02186

Ji, A. P., Drout, M. R., \& Hansen, T. T. 2019, arXiv:1905.01814

Kasen, D., Metzger, B., Barnes, J., Quataert, E., \& Ramirez-Ruiz, E. 2017, Natur, 551, 80

Kasliwal, M. M., Kasen, D., Lau, R. M., et al. 2019a, MNRAS, arXiv:1812. 08708

Kasliwal, M. M., Cannella, C., Bagdasaryan, A., et al. 2019b, PASP, 131, 038003

Kasliwal, M. M., Nakar, E., Singer, L. P., et al. 2017, Sci, 358, 1559

Lazzati, D., Perna, R., Morsony, B. J., et al. 2018, PhRvL, 120, 241103

LIGO Scientific Collaboration, Aasi, J., Abbott, B. P., et al. 2015, CQGra, 32, 074001

LIGO Scientific Collaboration and Virgo Collaboration 2019a, GCN, 24168, 1 LIGO Scientific Collaboration and Virgo Collaboration 2019b, GCN, 24237, 1

LIGO Scientific Collaboration and Virgo Collaboration 2019c, GCN, 24411, 1

LIGO Scientific Collaboration and Virgo Collaboration 2019d, GCN, 24277, 1

Margutti, R., Berger, E., Fong, W., et al. 2017, ApJL, 848, L20

Messick, C., Blackburn, K., Brady, P., et al. 2017, PhRvD, 95, 042001

Metzger, B. D., Martínez-Pinedo, G., Darbha, S., et al. 2010, MNRAS, 406, 2650

Mooley, K. P., Nakar, E., Hotokezaka, K., et al. 2018, Natur, 554, 207

Nitz, A. H., Dent, T., Dal Canton, T., Fairhurst, S., \& Brown, D. A. 2017, ApJ, 849,118

Oke, J. B., \& Gunn, J. E. 1982, PASP, 94, 586

Pian, E., D'Avanzo, P., Benetti, S., et al. 2017, Natur, 551, 67

Shappee, B. J., Simon, J. D., Drout, M. R., et al. 2017, Sci, 358, 1574

Siegel, D. M., Barnes, J., \& Metzger, B. D. 2019, Natur, 569, 7755

Singer, L. P., \& Price, L. R. 2016, PhRvD, 93, 024013

Smartt, S. J., Chen, T. W., Jerkstrand, A., et al. 2017, Natur, 551, 75

Soares-Santos, M., Annis, J., Brout, D., Chen, H.-Y., \& Dey, A. 2017 a, Blanco Imaging of the Southern Sky, NOAO Proposal

Soares-Santos, M., Holz, D. E., Annis, J., et al. 2017b, ApJL, 848, L16

Soares-Santos, M., Kessler, R., Berger, E., et al. 2016, ApJL, 823, L33

Tanaka, M., \& Hotokezaka, K. 2013, ApJ, 775, 113

Troja, E., Piro, L., van Eerten, H., et al. 2017, Natur, 551, 71

Valeev, A. F., Sokolov, V. V., Castro-Tirado, A. J., et al. 2019, GCN, 24317, 1

Veitch, J., Raymond, V., Farr, B., et al. 2015, PhRvD, 91, 042003

Wu, M.-R., Barnes, J., Martínez-Pinedo, G., \& Metzger, B. D. 2019, PhRvL, 122,062701 